\title{
Modal Propellant Gauging - Blue Origin Payload
}

\author{
Celestine Ananda, Nicholas Bartel, Sheila Franklin, Megan Janiak, Taylor Peterson \\ Affiliation (Physics, Carthage College) \\ Kenosha, WI, United States
}

\begin{abstract}
Since 2008, the Modal Propellant Gauging (MPG) team, consisting of multidisciplinary undergraduate researchers from Carthage College, has been developing and testing a fuel gauging system for use in microgravity environments. Using experimental modal analysis (EMA) techniques, the goal of the MPG project is to develop a flight ready technology that gauges fuel in microgravity environments by correlating the modal response of a 1-g equilibrium surface to the microgravity surface response at the same fluid fill level. The technology has been tested aboard parabolic flights via a manned parabolic flight payload. The payload consists of two propellant tanks and is designed to measure the modal response of each propellant tank to an injected white noise signal via piezoelectric sensors. Flight data shows that the MPG method can measure fuel with greater than or equal to $1 \%$ resolution at and below $50 \%$ fill levels. Under funding from the Wisconsin Space Grant Consortium during the summer of 2018, the MPG team made improvements to the Blue Origin New Shepard research flight rig which is manifested to fly in the second half of 2018.
\end{abstract}

\section{Introduction}

The Modal Propellant Gauging (MPG) project aims to develop a higher resolution fuel gauging method for microgravity environments compared to traditional fuel gauging methods. The majority of work on MPG has been done by students with the direction, guidance, and aid of Dr. Kevin Crosby. There are two specific sub-projects within this overarching initiative: one is for testing the technology on parabolic flights; the other is to test the original hypothesis for a longer period of time via a Blue Origin New Shepard Mission.

1.1. Program Carthage College undergraduate students have been developing the Modal Propellant Gauging technology since 2008. There have been multiple versions of the payload, each with additional features to further advance the accuracy of the technology. Students have flown with Dr. Kevin Crosby on parabolic flights sponsored by NASA until NASA discontinued their flight program. Current flight campaigns have been completed through the Zero-G Corporation.

1.2. Purpose Spacecraft lifetimes are limited primarily by propellant. The microgravity environment of space renders direct volume measurement using traditional buoyancy and level techniques ineffective. Indirect methods are used instead, however these methods typically incur considerable additional mass and expense to missions. Additionally, typical methods of propellant gauging suffer from decreasing accuracy as the tank empties, rendering massgauging accuracy lowest at the end of mission life when gauging accuracy is the most important. Low-gravity gauging technologies in wide use include those that depend on bookkeeping, equations of state, and thermal mass estimation. Bookkeeping methods employs ow rate monitors or, often, just assumptions about ow rates during thruster rings, to estimate the total amount of propellant consumed over time. Such methods accumulate error over time and therefore introduce growing uncertainties in the estimate of remaining propellant mass. Equation of State methods rely on the inference of the liquid volume fraction within a tank pressurized by an external pressurizing gas (usually, Helium) based on measurements of pressure and temperature and the application of the ideal gas law (or other appropriate equation of state) to the ullage gas. In the PVT approach, the tank is considered isothermal, and method accuracy depends in part on the validity of this assumption. As in the case of bookkeeping methods, propellant mass estimates derived from applications of PVT methods are increasingly inaccurate at lower 11 fractions. Thermal mass estimates rely on measurements of the temperature change induced in a propellant tank by external heating. Thermal response is compared to simulation results based on a tank-specic thermal capacitance model computed over a range of 11 fractions. The uncertainty of propellant mass estimates based on TPG methods depend sensitively on the sophistication 
and delity of the tank thermal model. In addition to low-gravity methods, settled propellant levels may be estimated using capacitance probes and various versions of impedance sensors. These electrical measurements are performed on settled liquids and are therefore used only during thrusting maneuvers and are subject to error induced by adhered liquid on sensors. Each family of methods has relatively reduced accuracy at low tank 11 fractions and therefore propellant gauging. Error estimates on most gauging methods range from $1 \%$ to $20 \%$ or more. Adding additional propellant mass to account for gauging errors can result in signicant launch costs and/or reduced mission life. In the work reported here, we introduce a complementary method of low-gravity propellant gauging that relies on real time modal analysis of acoustic resonances in a propellant tank. The Modal Propellant Gauging (MPG) approach has the advantage of increased accuracy at low propellant $1 \mathrm{l}$ fractions, is eective in both settled and sloshing propellants, and does not employ invasive hardware. Additionally, lab and parabolic ight testing suggest that the MPG method has gauging resolutions that are better than bookkeeping and PVT methods and comparable to the best TPG methods at low 11 fractions, with gauging resolutions approaching 1.0\% for settled propellant and 1.5-2.0\% for unsettled (sloshing) propellants.

The MPG project is a collaboration between Carthage Space Sciences and Kennedy Space Center Cryogenics Laboratory to develop a high-resolution, low-gravity, fuel gauging technology that exploits the effect of fluid loading on the structural properties of liquid-filled propellant tanks. The technology that has been developed will be implemented in the Orion/SLS Exploration Mission architecture. Because of the great interest in the project, a development of a smaller version of the propellant gauging device will be put onboard a Blue Origins rocket as a research payload.

1.3. Concept The concept behind the Modal Propellant Gauging technology is based on analyzing resonance frequencies of fluid-filled tanks. The surface of a liquid in microgravity does not settle as it would on Earth and conventional methods that are used on Earth to determine liquid levels are not effective. The configurations of liquid surfaces in microgravity environments are driven by tension and capillary forces. Because of this, liquid adheres to the inner walls of the fuel tanks in a microgravity environment. The Modal Propellant Gauging technique takes advantage of the variable thickness of the liquid level on the walls of a tank.

Experimental Modal Analysis (EMA) involves recording the vibration spectrum of a solid object and using the spectral characteristics to infer the structural properties of the object. Every object has natural response frequencies, normal modes, based on the geometry and mass of the object. The responsive frequencies of the fuel tanks lower as fluid is added to the tanks. As fluid is added the thickness of the liquid layer adhered to the tank walls increases. The thicker the liquid layer on the walls, the lower the frequency response to vibration will be. We observe the response frequencies of the tanks and their constituent liquid mass between $600 \mathrm{~Hz}$ and $1000 \mathrm{~Hz}$. We do not observe higher frequency responses because of substantial increases in electrical noise. This technique works most successfully at lower fill levels because after increasing the amount of fluid substantially, the inner walls are essentially coated.

The EMA technique requires that we stimulate all acoustic resonances in the tank structure by applying a broadband white noise signal. Tank response is measured at discrete locations on the surface of the tank and the Fourier Transforms of the response and input functions are computed in real-time. Finally, the Frequency Response Function (FRF) for each structural response is computed by essentially taking the ratio of the response FFT to the input FFT. The resulting FRF is a complex-valued function in which the real part is a function of the effective mass of the vibrating object and the imaginary part is related to the rate of energy dissipation through structural damping. Adding fluid to a tank lowers the real part of the frequency by increasing the effective mass. The resulting FRF with some noise filtering is shown below in Figure 1. As can be seen in Figure 1 the FRF amplitude peaks shift to to lower frequencies as the tank is filled.

\section{Parabolic Flights}

To determine the accuracy of the technique in microgravity environments, the MPG payload has been manifested to fly in a modified Boeing-727 with parabolic flight trajectories that simulate microgravity during the free-fall portion of the parabola. Each parabola allows data to be taken for nearly 20 seconds of microgravity. Following each portion of microgravity is a period of $2 \mathrm{~g}$ as the aircraft pulls up to climb the height it fell through. The current Carthage College Microgravity Team has been selected for funding to fly on multiple parabolic flight campaigns. 
2.1. Experimental Payload An image of the experimental payload can be seen in Figure 2. The frame of the payload was constructed using 10 series 80/20 aluminum t-slots, and secondary containment is maintained by gasketed polycarbonate panels on all sides that sit inside the recession of the t-slots. A hinged door at the top of the payload allows access to the interior of the payload. The payload contains two acrylic tanks connected by a fluid flow loop that allows for automated filling and draining of, and fluid transfer between, each vessel/propellant tank. Visual confirmation of the fluid levels in each tank is maintained through the use of two liquid level gauges whose bottoms are flush with the bottom of each tank. Both tanks have a set a four piezoelectric patch sensors adhered to their outer walls. The top two sensors are used for data collection, the bottom sensor is the actuator which inputs white noise to the system, and the second patch to the bottom is a monitor, which is used to remove actuator noise from the sensor data.

Data acquisition, signal control, and flow loop control are all maintained by a National Instruments 9133 data acquisition (DAQ) system. The data acquisition system sits in the interior of the payload between the two propellant tanks. An external interface is maintained through a touch screen monitor mounted to the top of the payload. All controls are run from the monitor through LabView. Accompanying the monitor on the top of the payload is an electronics box which contains all necessary components to regulate power to each electrical system.

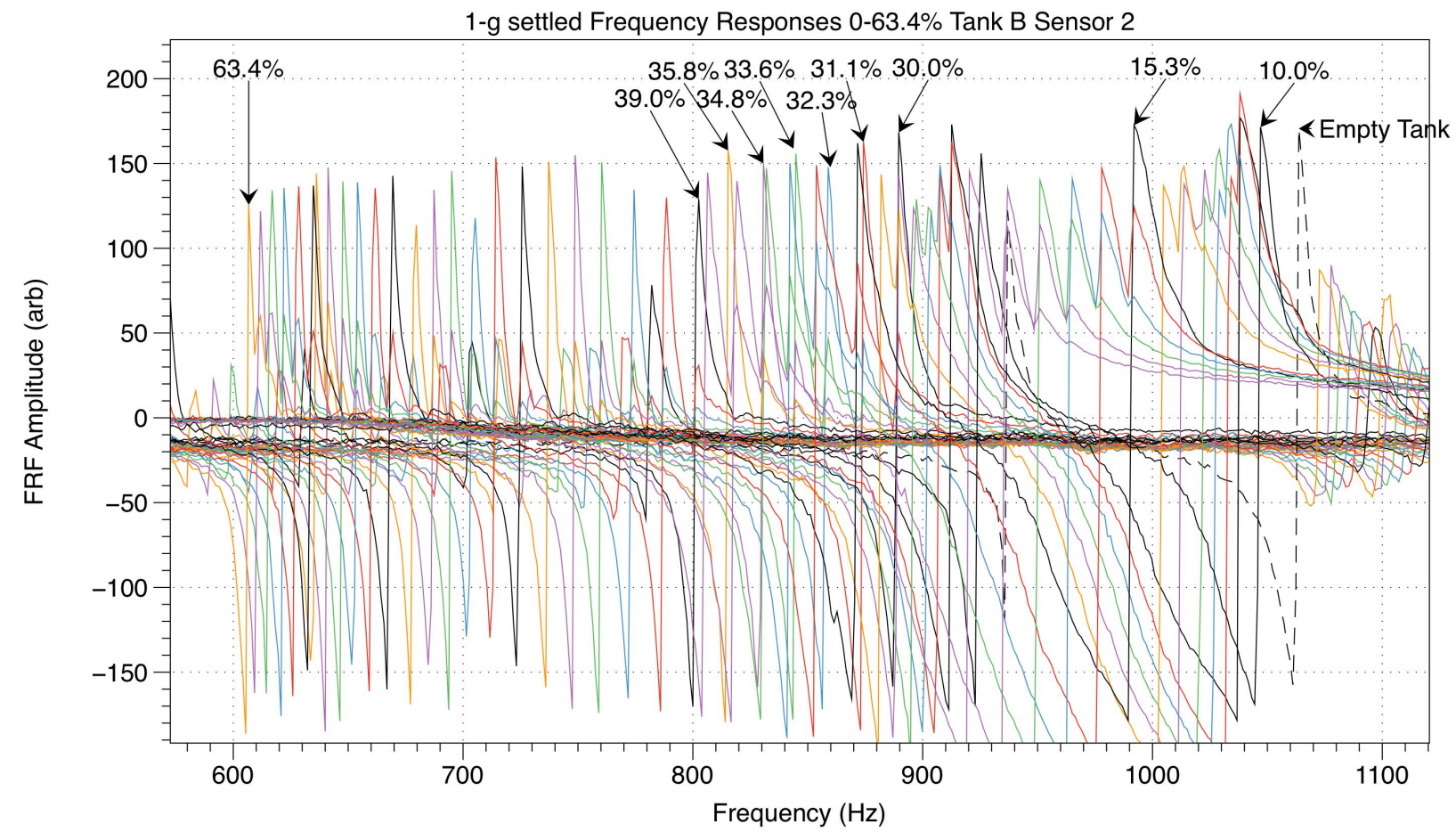

Figure 1: Frequency responses of a $1 \mathrm{~g}$ equilibrium surface

2.2. Rig Improvements Prior data has suggested that during the parabolic flights the fluid does not settle by the end of each period of micro-gravity. To have visual confirmation of this occurrence, the Composite Over-wrapped Pressure Vessel (COPV) tanks previously used were replaced with clear acrylic tanks. The acrylic tanks were designed to be exactly the same size as the COPV tanks. This prevented the need for a redesign of the flow loop or any of internal layout.

2.3. Data Collection Process The data collection process during the parabolic flights is simple because it is a manned payload. Those who fly with the experiment must activate the software at the beginning of each parabola. The flight crew informs the passengers when the aircraft is "pushing over", which signifies the start of the zero-g period. The crew then activates the software by pushing a button on the touchscreen to record data for the next 20 seconds of microgravity. The zero-g periods are not perfect, there are often negative impulses which cause the liquid in the tanks to slosh around. To accommodate this problem, the data for any given fill level is averaged over parabolas. After 5 


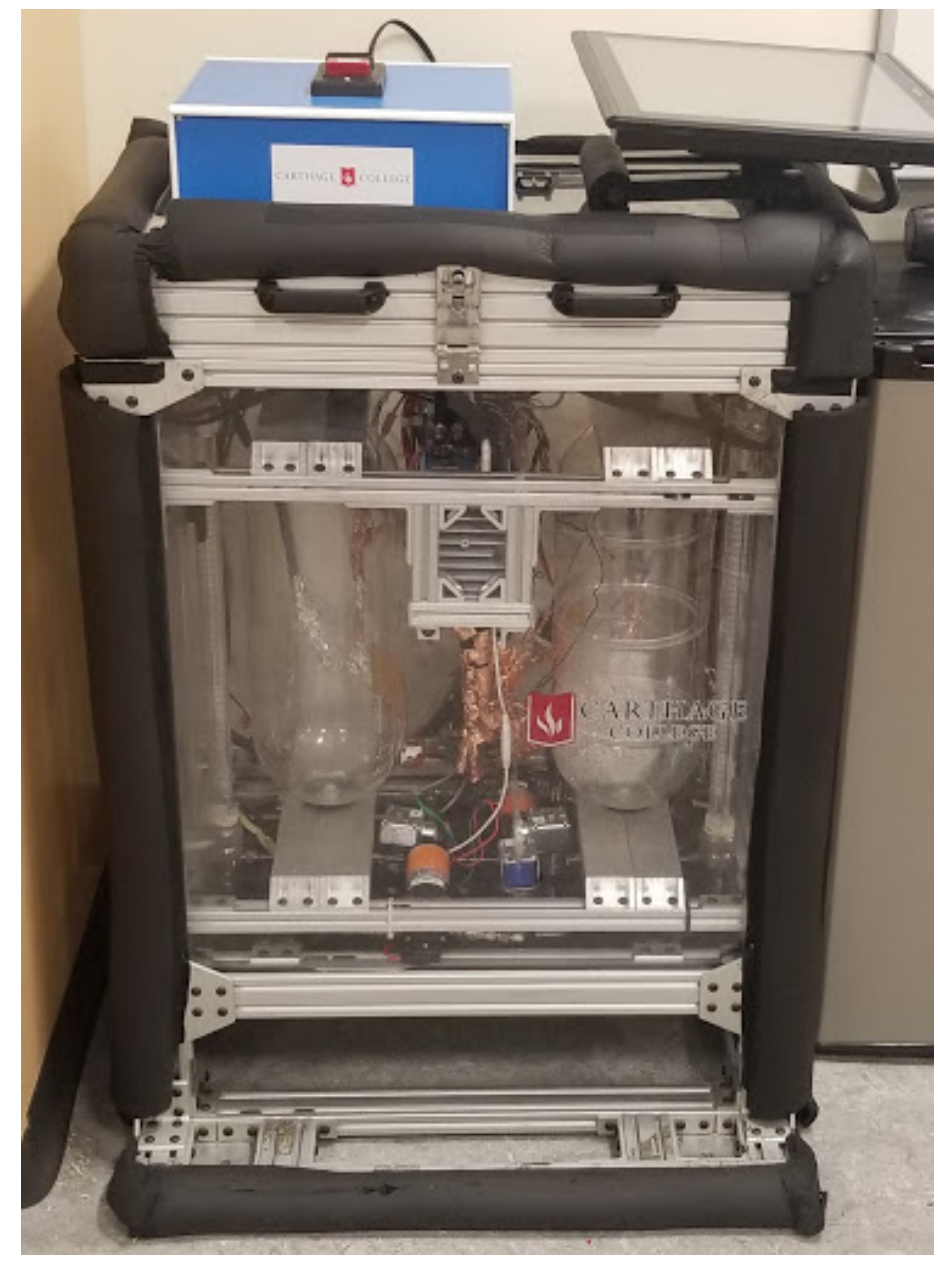

Figure 2: Parabolic flight payload

parabolas, the fill level is changed either during a 2- $g$ pull up or a 1- $g$ rest period. Testing is then resumed for the next set of parabolas. This extra sloshing does not allow the liquid inside the tanks to fully settle and coat the inner walls as desired. Because of this we have implemented three concentric rings attached to the inner walls of the tanks to get more realistic results. It is important to note that the fill levels are approximated by the liquid level gauges that are a part of the flow loop of the experimental rig.

2.4. Analysis The image in Figure 1 is a sample of data collected from a single tank in 1-g over a range of an empty tank to fill level of nearly 50 percent of the tank volume. The technique is able to recognize a one percent change of total volume based on the modal response of the tank. This data was taken in our lab; while aboard the aircraft there is substantial noise in the data due to fluid sloshing which decreases the resolution. 


\section{New Shepard Payload}

3.1. New Shepard Introduction The success of the Modal Propellant Gauging technique has garnered substantial interest from NASA for future missions to Mars, as the technique cuts down on costs substantially due to the lightweight nature of the sensors. However, NASA has concerns that the fluid in the tanks may reach equilibrium in different configurations. Our simulations show that this will not occur, and that the fluid will consistently coat the walls of the tanks evenly, not settling only on one side of the tank or in any one location. The parabolic flights allow for 20 seconds of zero-gravity, which is not enough time to reach equilibrium. Our simulations have shown that it should take around 1 minute and 30 seconds to reach equilibrium, varying based on initial applied forces, but should not take longer than 2 minutes. Because of this, a smaller, modified version of our payload has been selected for manifestation aboard a Blue Origin New Shepard vehicle, allowing for 3 minutes of zero-gravity. This test should alleviate NASA's concerns with the technique by providing video evidence of how the fluid will settle in the tanks.

Blue Origin's New Shepard is a re-usable, sub-orbital launch vehicle designed to transport a six-person crew to the region beyond Earth's lower atmosphere. During manned flights, the crew will experience the journey inside a crew capsule located atop the vehicle. The research flight our payload will fly aboard is an unmanned flight, and the crew capsule will contain payload lockers in place of seats to hold several experiments.

3.2. Payload Concept The team took an "inside-out" design approach for the New Shepard payload, where the payload was designed from the propellant tanks outward. Fluid properties do not scale linearly, so given the dimensional constraints of the payload locker, careful consideration was given to determine the propellant tank size. If the tanks are too small, the properties of the fluid, as well as the fluid dynamics, deviate from an accurate re-creation of in-flight conditions. If the tanks are too large, the number of propellant tanks contained within the payload must decrease, which in turn reduces the amount of data we could record. Furthermore, it was important to consider not only the absolute dimensions of the tanks, but the ratio of the height of the tanks to their diameter (cylindrical tanks were used). After running computational fluid dynamics (CFD) simulations, the dimensions of the tanks were chosen to be 10.8 " in height and 6 " in diameter. Given the size of the propellant tanks, water will be used as the model propellant to preserve the scaling properties of the fluid dynamics. The remaining elements of the payload were designed around the size and arrangement of the tanks, and the overall structure was designed to use all of the available interior volume provided by the payload locker.

Unlike the parabolic flight payload, the New Shepard payload will use only one sensor and a monitor, rather than two sensors and a monitor. Additionally, there is no flow loop in the New Shepard payload, so each tank will contain a different, yet fixed fill level. Lastly, the experiment will run autonomously, collecting data throughout the entire duration of the flight. Overall, the payload was designed to be as simple as possible in mechanical and electrical design, as well as concept. Because this will be the first New Shepard flight for the Modal Propellant Gauging team, it was decided that a simpler payload would increase the chance of mission success.

3.3. Payload Design and Construction The payload was made a rectangular cube of nominal dimensions 18 " $\times 14 " \times 18$ ". The structural frame of the payload was constructed using 10-series $80 / 20$ aluminum t-slots, and the t-slots are held together by 80/20 aluminum joining plates that use bolts and t-nuts. A labeled diagram of the payload can be found in Figure 3. 


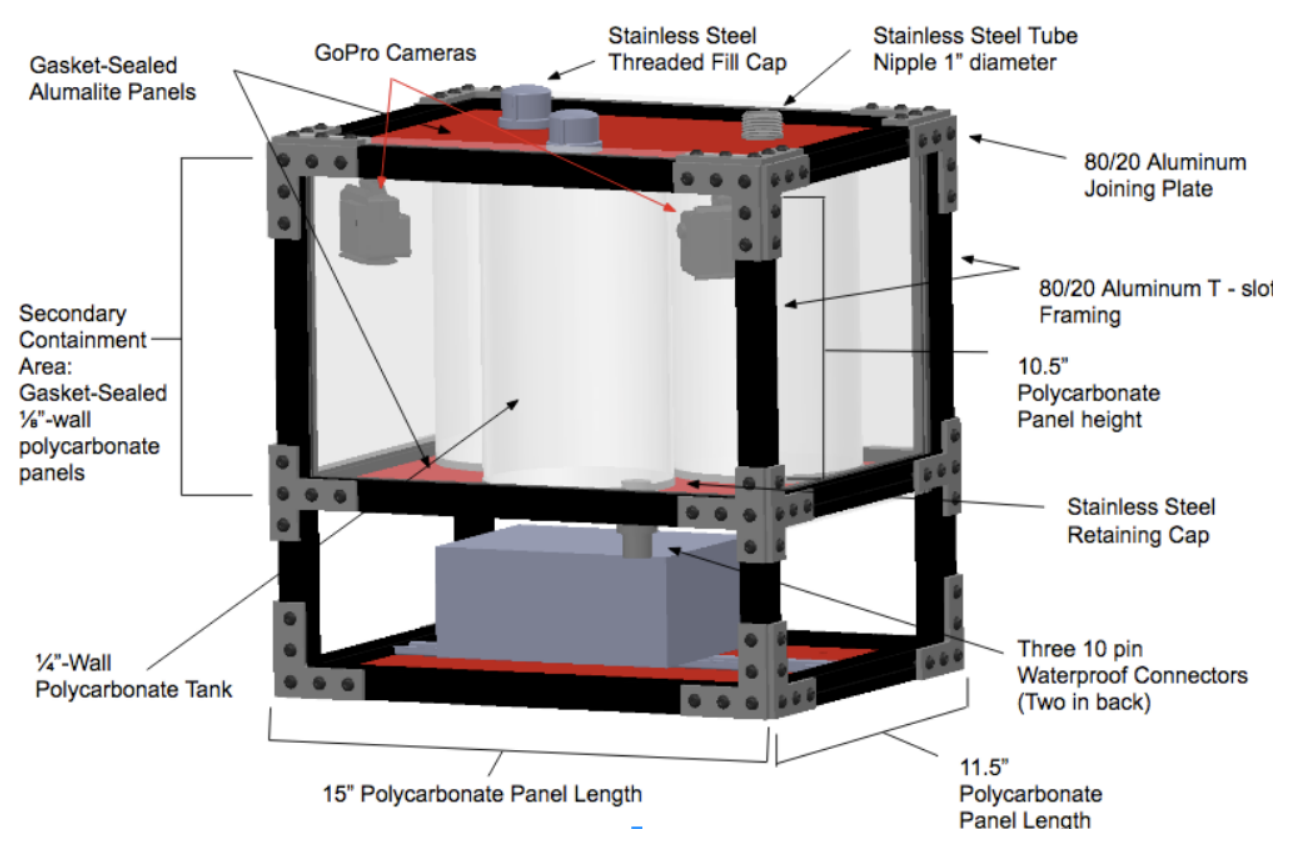

Figure 3: Labeled Solidworks model of New Shepard payload

The payload is divided into two sections: the secondary containment area (top), and the electronics and mounting deck (bottom).

3.3. Secondary Containment Area The Secondary containment area is kept sealed via four gasketed polycarbonate panels on each side, two gasketed Alumalite panels on the top and bottom, and three threaded stainless steel pipe nipples with sealant applied around the base of the nipples where they protrude from the secondary containment area. The pipe nipples allow external access to the interiors of the tanks, but not to the interior of the secondary containment area. Contained within the secondary containment area are three polycarbonate cylindrical propellant tanks arranged in an isosceles triangular formation, cylindrical propellant tank mounting caps, and two GoPro action cameras mounted in the front top corners of the containment area and angled downward and inward at approximately 45-degree angles to allow for a full view of all three propellant tanks. Visual confirmation of the settled state of the propellant is one of the success criteria of the mission, so the secondary containment area will also contain LED lighting strips for interior lighting.

3.3. Electronics and Mounting Deck The data acquisition system, power regulators, white noise generators, and signal amplifiers will all sit inside an aluminum project box mounted to the payload deck via a din rail. All required electrical connections will be wired into the secondary containment area via a hermetically sealed bulkhead connector that penetrates the secondary containment area from the bottom at the back of the payload. The payload will be mounted to the locker by bolts directly through the electronics and mounting deck into the threaded holes of the locker's grid pattern. Eight bolts will be used in total.

3.3. Construction The payload was initially drafted in Solidworks for verification of dimensions and proof of concept. After the design of the payload was finalized, construction began. Leading up to the research flight in the first half of 2018, construction of the payload will be completed along with full systems integration, full mission simulations, and integration of the payload into the payload locker system. Figure 4 displays an labeled diagram of the payload. 


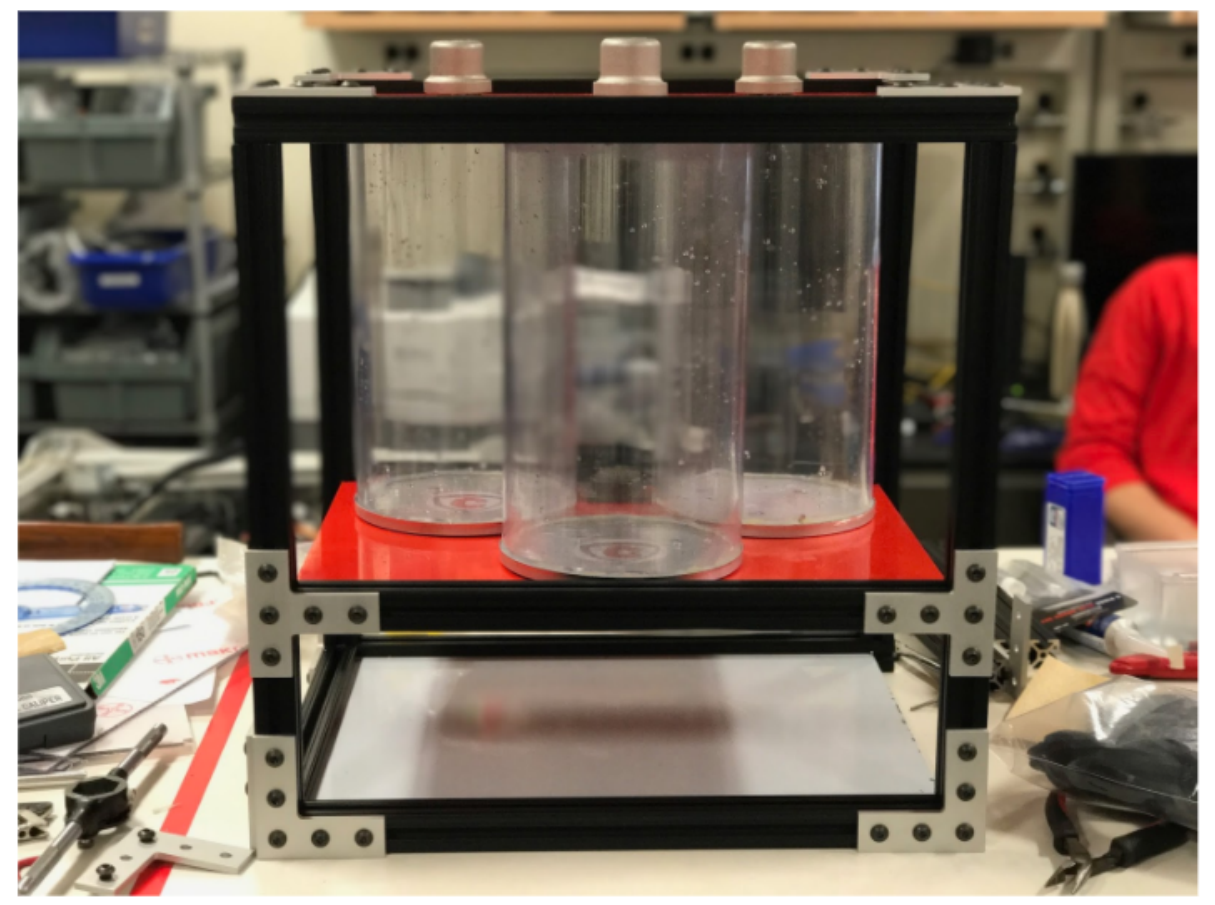

Figure 4: New Shepard payload

\section{Summary}

The MPG project is a collaboration between Carthage Space Sciences and Kennedy Space Center Cryogenics Laboratory to develop a high-resolution low-gravity fuel gauging technology that exploits the effect of fluid loading on the structural properties of liquid-filled propellant tanks. The technology that has been developed will be implemented in the Orion/SLS Exploration Mission architecture. A concern with the technique is that the fluid may not reach the same equilibrium configuration during every use which would drastically depreciate the accuracy of the technique. Our simulations show that this will not occur, but to alleviate this concern a smaller version of the propellant gauging device is being developed to put onboard a Blue Origin New Shepard rocket as a research payload. This test will allow for more than enough time to reach equilibrium, helping to finalize the implementation of the technique in the Orion/SLS Exploration Mission.

\section{Acknowledgments}

We would would to thank our advisor and the director of WSGC, Kevin Crosby, for giving us the opportunity to work on this project. We would additionally like to thank the Wisconsin Space Grant Consortium for funding this project. Finally, we would like to thank the Zero-G corporation for allowing us the opportunity to test our project in a microgravity environment. 